\title{
Composite metamaterial square grids with sign-flipping expansion coefficients leading to a type of Islamic design
}

\author{
Teik-Cheng $\operatorname{Lim}^{1} \mathbb{D}$
}

Received: 25 February 2020 / Accepted: 7 April 2020 / Published online: 17 April 2020

(c) Springer Nature Switzerland AG 2020

\begin{abstract}
A class of 2D metamaterials with sign-switching coefficients of thermal, pressure and moisture expansions is proposed herein-such materials not only change properties with environmental changes, but also reverses between positive and negative. Designed from bimaterial strips and arranged into the form of square grids, changes to environmental conditions transform the square grids into 2D array of 8-pointed stars found in Islamic art and architecture. Results indicate that the bimaterial strip deflection can fit the 8-pointed star when the overall strain is-0.105. Suppose the general expansion coefficients for both materials in the bimaterial strip $\left(\alpha_{1}, \alpha_{2}\right)$ are known, then the required environmental changes for attaining the 8-pointed star array can be determined. The currently proposed metamaterial not only demonstrates its capability of attaining properties with reversible sign, but also achieve aesthetic feature with historical significance.
\end{abstract}

Keywords Environmental change $\cdot$ Islamic art · Metamaterials · Sign-toggling

\section{List of symbols}

$\alpha \quad$ Generic coefficient of environmental expansion

$\alpha_{1} \quad$ Generic coefficient of environmental expansion for material 1 of bimaterial strip

$\alpha_{2} \quad$ Generic coefficient of environmental expansion for material 2 of bimaterial strip

$\alpha_{v}^{(C)} \quad$ Volumetric coefficient of moisture expansion

$\alpha^{(C)}$ Linear coefficient of moisture expansion

$\alpha_{v}^{(T)} \quad$ Volumetric coefficient of thermal expansion

$\alpha^{(T)} \quad$ Linear coefficient of thermal expansion

$\alpha_{V}^{(P)}$ : Compressibility, or the coefficient of compressibility

$\alpha^{(P)} \quad$ Linear coefficient of compressibility

$\varepsilon_{C} \quad$ Strain due to change in moisture concentration

$\varepsilon_{P} \quad$ Strain due to pressure change

$\varepsilon_{T} \quad$ Strain due to temperature change

$C$ Moisture concentration

$E_{1} \quad$ Young's modulus of material 1 in bimaterial strip

$E_{2} \quad$ Young's modulus of material 2 in bimaterial strip

$h \quad$ Thickness of bimaterial strip $h_{1} \quad$ Thickness of material 1 in bimaterial strip

$h_{2} \quad$ Thickness of material 2 in bimaterial strip

$l_{1}$ Second moment area of material 1 in bimaterial strip

$I_{2}$ Second moment area of material 2 in bimaterial strip

$K \quad$ Bulk modulus

$L \quad$ Generalized length

I Length of a segment of bimaterial

$m \quad$ Moisture mass in solid

$M \quad$ Mass of dry solid

$P \quad$ Pressure

$r \quad$ Radius of curvature of a bimaterial due to environmental change

$T$ Temperature

$v \quad$ Volume

Teik-Cheng Lim, alan_tc_lim@yahoo.com | 'School of Science and Technology, Singapore University of Social Sciences, Singapore, Singapore. 


\section{Introduction}

Metamaterials are artificially-designed materials possessing characteristics that do not exist naturally. Such physical behavior arise from the architectured microstructure and not due to the base material properties. The term metamaterial is derived from a Greek word $\mu \varepsilon t a ́$ (meta), which means beyond, because they exhibit properties beyond those that are found naturally. Of late, metamaterials have proven their capabilities to achieve great practical importance. For example, Tümkaya et al. [1] developed an efficient metamaterial-based portable fuel oil sensor in order to distinguish branded fuel-oil samples from unbranded ones, while Abdulkarim et al. [2] proposed a broadband coplanar waveguide (CPW)fed monopole antenna based on conventional CPW-fed integration with an organic solar cell (OSC) of $100 \%$ insolation for $\mathrm{K}_{\mathrm{u}}$ band satellite communication. This configuration was designed to permit $100 \%$ insolation of the OSC, thereby enhancing the performance of the antenna. In the investigation by Ozdemir et al. [3], a resonator layer was suggested for reducing the mutual coupling effect between each antenna element of a cross dipole antenna. The results were noted for improving isolation in multiport antenna systems, based on artificial neural network approach. A new metamaterials-based hypersensitized liquid sensor integrating omega-shaped resonator with microstrip transmission line was proposed by Abdulkarim et al. [4]; this metamaterial integrated transmission line-based sensor can be a potential candidate for precise detection of fluidics and for applications in the fields of medicine and chemistry. In addition, Dalgac et al. [5] designed a chiral metamaterial-based sensor for detecting the variation in substance ratio of chemical substances when combined with distilled water. Their investigation provides distinctive sensing results and therefore avails a novel method to the microfluidic sensor applications by using the sample holder.

Although negative material properties-such as negative Poisson's ratio [6-22], negative thermal expansion [23-31], negative compressibility [32-37] and negative moisture expansion [38-40] - have been investigated for a few decades due to their capability of behaving in ways that are not achievable by conventional materials, it remains that some applications may require both positive and negative properties depending on the circumstances. For this reason, a series of metamaterials in which their effective material properties can switch between positive and negative signs have been introduced. These include the change in the Poisson's ratio sign upon reversal of stress direction $[41,42]$, the change in the coefficient of thermal expansion sign upon temperature fluctuation $[43,44]$, the change in Poisson's ratio sign with temperature fluctuations $[45,46]$, and negative Poisson's ratio metamaterial with sign-switching expansion coefficients with environmental fluctuations $[47,48]$.

While the above-mentioned metamaterials exhibit properties with sign-toggling capabilities in order to suit the changing loading and/or environmental condition, the artistic aspects are lacking. Inspired by Islamic art, Rafsanjani and Pasini [49] developed a set of metamaterial microstructures that manifest negative Poisson's ratio behaviour. In this paper a class of square grids made from bimaterial strips that transforms into arrays of 8-pointed stars is introduced. The array of 8-pointed stars, as exemplified in Fig. 1, is prevalent in Islamic art and architecture. The motivation of choosing this design, therefore, is not only confined to the potential for creating metamaterials with physical properties that switch between positive and negative values by environmental changes and without any active control, but also to pay homage to an ancient art that is associated with a great religion. This geometry is also chosen because the art forms are hidden at the reference environmental condition and are only manifested when the environmental condition changes, thereby creating a dynamic micro-lattice structure. As illustrated in Fig. 2, this paper proposes a 2D metamaterial in which the increase in temperature $d T>0$ and/or moisture concentration $d C>0$ as well as decrease in pressure $d P<0$ lead to material contraction, thereby exhibiting negative expansivity. However, the decrease in temperature $d T<0$ and/or moisture concentration $d C<0$ as well as increase in pressure $d P>0$ also lead to material contraction, thereby exhibiting positive expansivity instead.

\section{Analysis}

Two configurations of bimaterial strips within the square grids are identified and illustrated in Figs. 3 and 4 to demonstrate the manner at which the bending of the straight bimaterial strips in response to changing environmental condition can transform the square grids into arrays that approximate 8-pointed stars found in Islamic art. Specifically, we term the configurations furnished in Figs. 3 and 4 as the Type A and Type B, respectively. The bimaterial strips in both types consist of two materials, with material 1 (indicated by yellow) possessing a higher expansion coefficient than material 2 (indicated by green), such that different extent of expansion leads to materials 1 and 2 being convex and concave, respectively, as shown in Fig. 3 (right) and Fig. 4 (right). Conversely, under an opposing change in environmental condition, the 

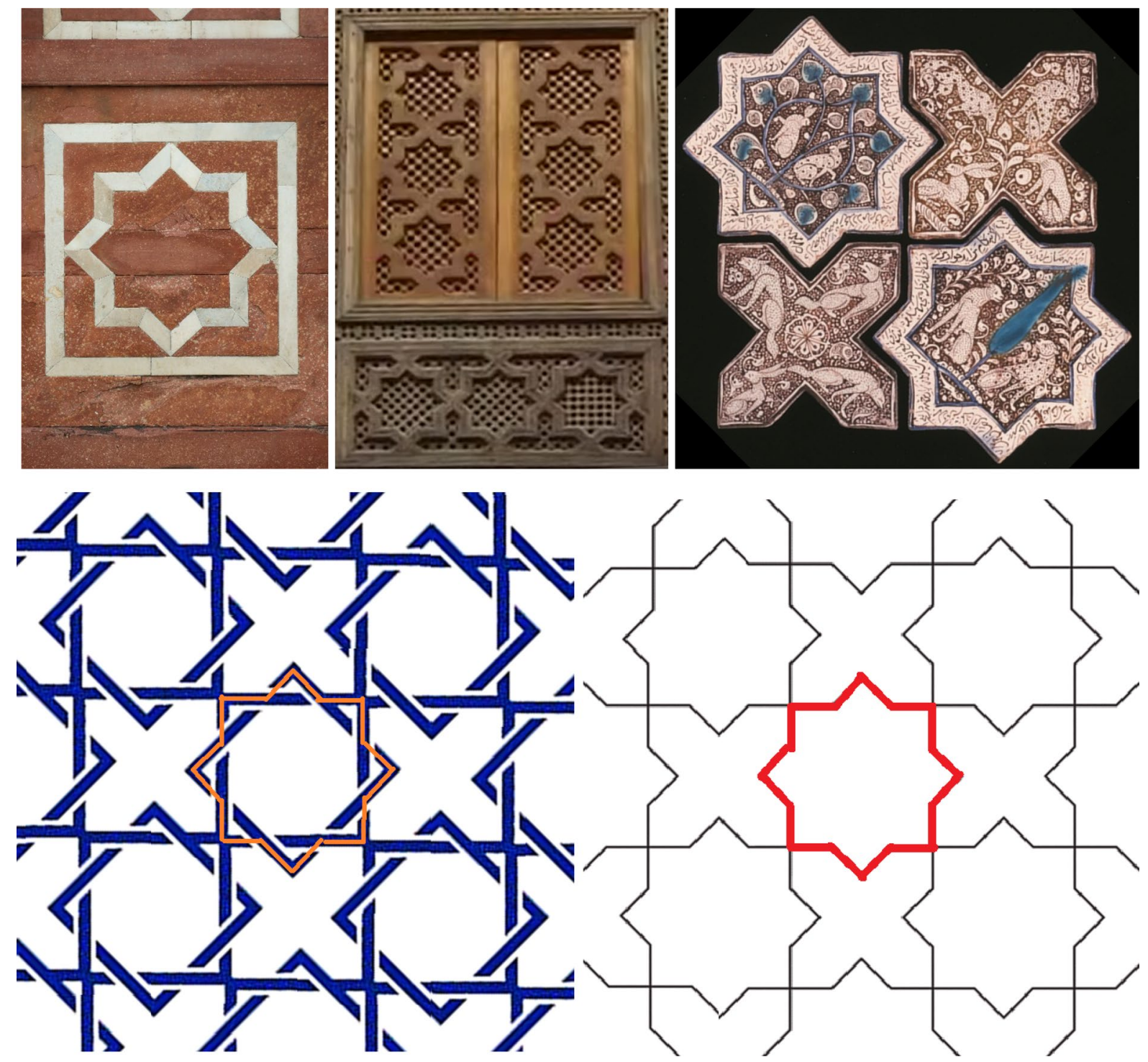

Fig. 1 Examples of Islamic 8-pointed connected star: a single star in sixteenth century Humayun's tomb, India (top left), a few stars arranged vertically and horizontally in an interior part of the fourteenth century Al-Attarine Madrasa, Egypt (top centre), stars

differential contraction leads to materials 1 and 2 being concave and convex, respectively, as furnished in Fig. 3 (left) and Fig. 4 (left). In both Type A and Type B metamaterials, one end of each bimaterial strip is built into rigid squares (shown in red) and each square is attached to four bimaterial strips. Unlike Type B, the configuration in Type A permits free rotation at the other end of each bimaterial strip, which is attached to a pillow block bearing (indicated in blue) such that the curved outer surface of each pillow bearing is in contact to another pillow block bearing via connectors (indicated in black) through the holes. Unlike Type A, each bimaterial strip in Type $B$ consists of three segments with length ratio 1:2:1 arranged diagonally in tiles from thirteenth century Iran (top right), overlapping design (bottom left), and simplified schematics (bottom right)

whereby materials 1 and 2 alternate from one segment to the next.

The bimaterials are permitted to curve based on three types of environmental changes-temperature, pressure and moisture concentration-through contrasting (i) coefficient of thermal expansion (CTE), (ii) compressibility, or coefficient of pressure expansion (CPE), and (iii) coefficient of moisture expansion (CME). The volumetric CTE for any matter-be it in the form of solid, liquid or gas-is a measure of the volumetric change in response to temperature change at constant pressure, and is expressed as 
Fig. 2 Transformation of a square grid (top) to an 8-pointed star array under a change of environmental condition (bottom left) and its conjugate form under an opposing change of environmental condition (bottom right)

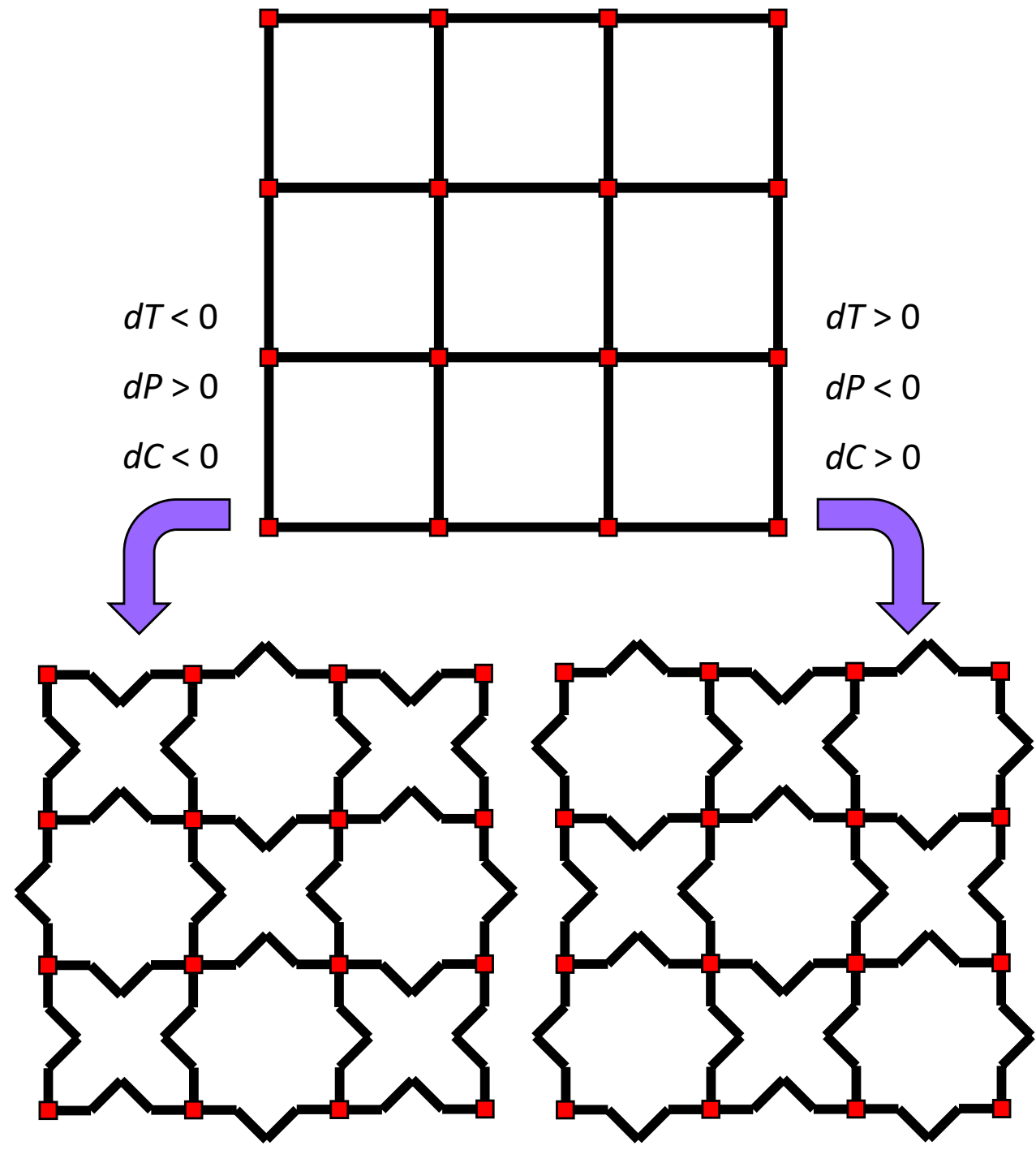

$\alpha_{V}^{(T)}=\frac{1}{V}\left(\frac{\partial V}{\partial T}\right)_{P}$

Due to anisotropy, which is common in solids, it is more meaningful to write separate CTEs in each orthogonal direction. Hence we have the linear CTE

$\alpha^{(T)}=\frac{1}{L} \frac{d L}{d T}=\frac{\varepsilon_{T}}{d T}$

where $\varepsilon_{T}$ is thermal strain. In thermofluid mechanics, the coefficient of compressibility, or more conveniently the "compressibility", quantifies the volumetric change in response to pressure change. When these changes take place at constant temperature, we have the isothermal compressibility

$$
\alpha_{V}^{(P)}=-\frac{1}{V}\left(\frac{\partial V}{\partial P}\right)_{T}
$$

The negative sign indicates a decrease in size with an increase in pressure. For the purpose of consistency with the linear CTE, we herein introduce its linear version as the CPE

$\alpha^{(P)}=-\frac{1}{L} \frac{d L}{d P}=\frac{\varepsilon_{P}}{(-d P)}$

where $\varepsilon_{p}$ is the strain due to pressure change. To obtain an analogous coefficient due to moisture absorption into or moisture dissipation from a solid, we recall the definition of moisture concentration in solids

$$
C=\frac{m}{M} \times 100
$$


Fig. 3 Type A square grid in its original state (top) and its deformation approximating the 8-pointed star under opposing conditions that lead to opposing curvatures in the bimaterial strips (bottom), where the material 1 (yellow) has greater expansion coefficients than material 2 (green)

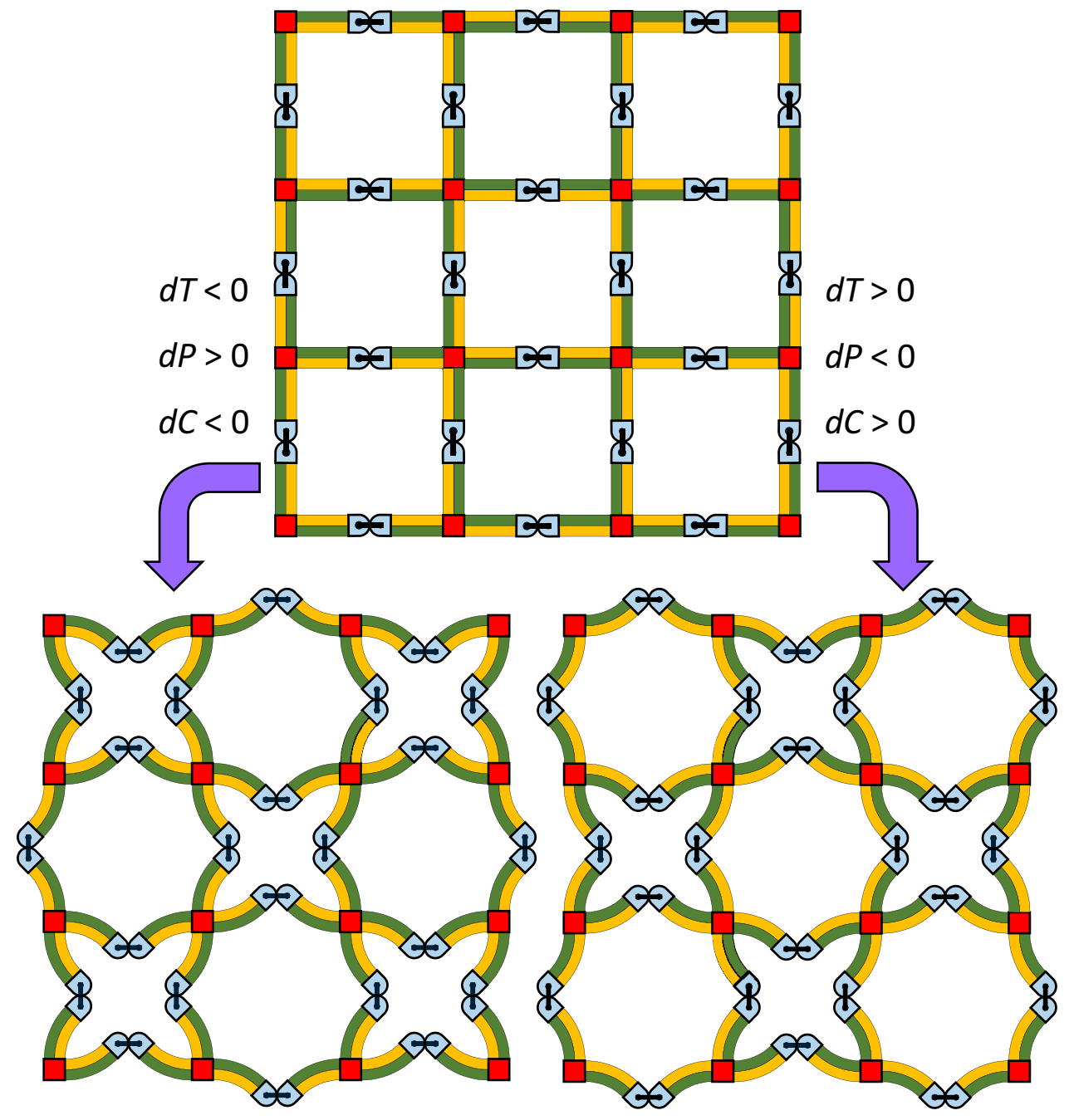

where $m$ is the mass of water and $M$ is the mass of the dry material, hence the change in moisture concentration is

$d C=\frac{d m}{M} \times 100$

where $d m$ is the change in moisture mass in the solid. This also applies for the environmental moisture concentration wherein $d m$ is the change in moisture mass per unit volume of the environment while $M$ refers to the mass of dry air in the same volume. Therefore the CME due to the change in moisture concentration in the solid is

$\alpha^{(C)}=\frac{1}{L} \frac{d L}{d C}=\frac{\varepsilon_{C}}{d C}$

where $\varepsilon_{C}$ is the strain arising from the change in moisture concentration. Due to different absorptivity level, various materials absorb differing amount of moisture from the environment. As such, a change in environmental moisture concentration $d C$ leads to changes in moisture concentration in materials 1 and $2\left(d C_{1}, d C_{2}\right)$ in a twophase composite wherein $d C \neq d C_{1} \neq d C_{2}$ at moisture transfer equilibrium. This is unlike temperature change where $d T=d T_{1}=d T_{2}$ is attained at thermal equilibrium [40]. Analogies can be formed with the various coefficients of expansion and their corresponding strains and environmental changes, as listed in Table 1.

Recall that for a straight bimaterial strip with materials 1 and 2 possessing CTEs of $\alpha_{1}^{(T)}$ and $\alpha_{2}^{(T)}$, respectively, the radius of curvature $r^{(T)}$ that is formed under a temperature change of $d T$ is [50]

$\frac{1}{r^{(T)}}=\frac{\left(\alpha_{1}^{(T)}-\alpha_{2}^{(T)}\right) d T}{\frac{h}{2}+\frac{2}{h}\left(E_{1} l_{1}+E_{2} I_{2}\right)\left(\frac{1}{E_{1} h_{1}}+\frac{1}{E_{2} h_{2}}\right)}$

where

$h=h_{1}+h_{2}$ 
Fig. 4 Type B square grid in its original state (top) and its deformation approximating the 8-pointed star under opposing conditions that lead to opposing curvatures in the bimaterial strips (bottom), where the material 1 (yellow) has greater expansion coefficients than material 2 (green)

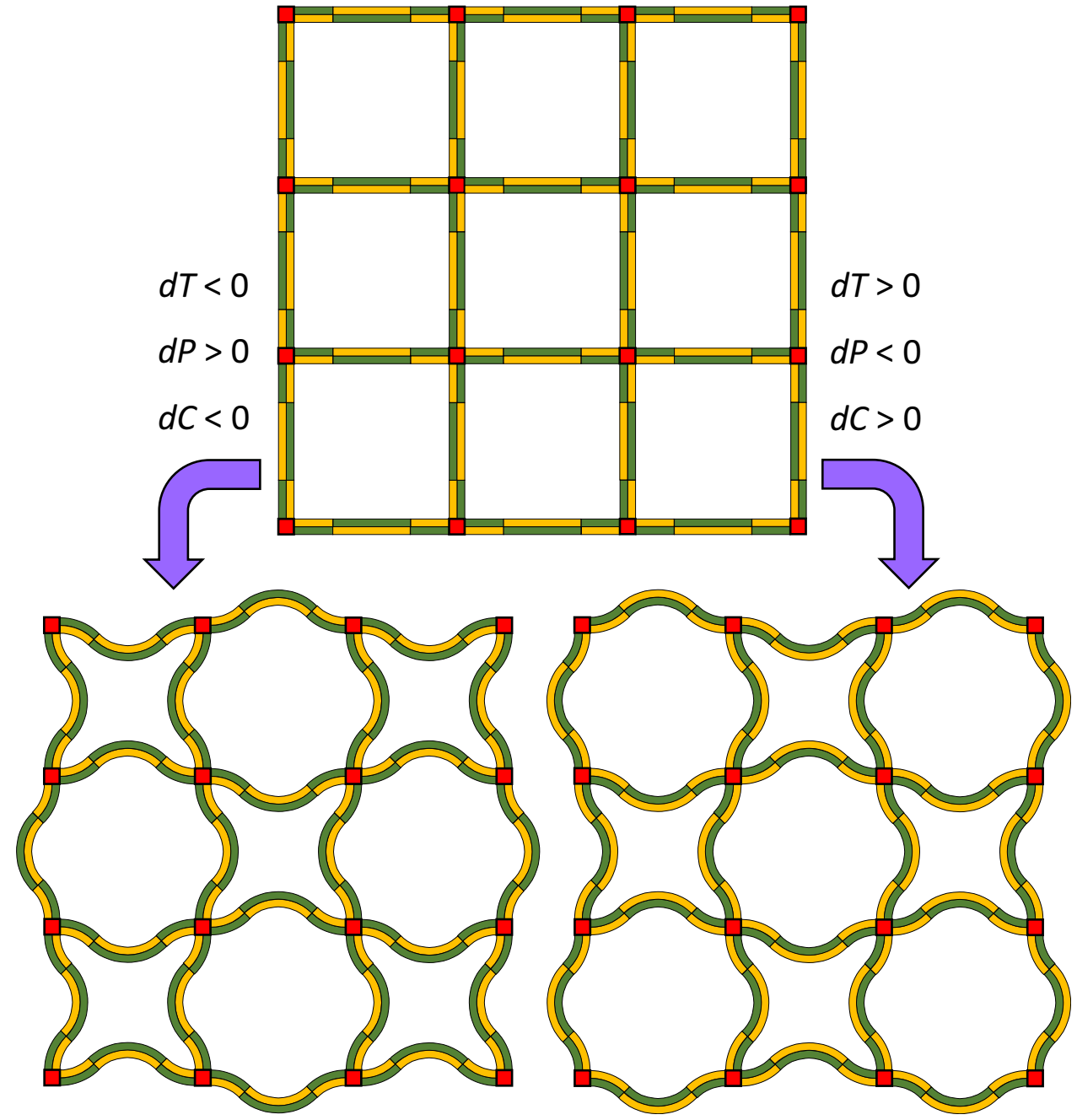

Table 1 Analogies between specific coefficients of environmental expansions and related parameters

Thermal

Pressure

Moisture concentration

\begin{tabular}{llll}
\hline Environmental change & $d T$ & $-d P$ & $d C$ \\
Strain response & $\varepsilon_{T}$ & $\varepsilon_{P}$ & $\varepsilon_{C}$ \\
Expansion coefficient & $\alpha^{(T)}$ & $\alpha^{(P)}$ & $\alpha^{(C)}$ \\
\hline
\end{tabular}

and

$I_{1}=\frac{h_{1}^{3}}{12}, \quad I_{2}=\frac{h_{2}^{3}}{12}$

with $h_{1}$ and $h_{2}$ being the thicknesses of materials 1 and 2 , respectively. By similar reasoning on the differential expansion of the bonded strips due to pressure change $d P$, the resulting curvature $r^{(P)}$ is
$\frac{1}{r^{(P)}}=\frac{\left(\alpha_{1}^{(P)}-\alpha_{2}^{(P)}\right)(-d P)}{\frac{h}{2}+\frac{2}{h}\left(E_{1} I_{1}+E_{2} I_{2}\right)\left(\frac{1}{E_{1} h_{1}}+\frac{1}{E_{2} h_{2}}\right)}$

where $\alpha_{1}^{(P)}$ and $\alpha_{2}^{(P)}$ are the CPEs of materials 1 and 2, respectively, of the bimaterial strip. As mentioned earlier, the negative sign in Eq. (4) implies the decrease in dimension with increase in pressure; this results in the negative sign in Eq. (11), which indicates that the bimaterial strip curves in the opposite direction with increasing pressure when compared to the case of increasing temperature. While the temperature change in bimaterial strip is equal to that in the environment at thermal equilibrium $d T_{1}=d T_{2}=d T$, and that the pressure change experienced by the bimaterial strips are common to the pressure change in the environment $d P_{1}=d P_{2}=d P$, the same cannot be said so for the case of moisture change. Due to different level of moisture concentration change between the environment and materials even at hygroscopic equilibrium, as well as the different extent of moisture retention in each material, 
the change in moisture concentration at hygroscopic equilibrium for the environment and in both materials in the bimaterial strip are different. As a result of the different coefficients of moisture expansion $\left(\alpha_{1}^{(C)}, \alpha_{2}^{(C)}\right)$ and different extent of moisture concentration change at hygroscopic equilibrium $\left(d C_{1}, d C_{2}\right)$ in materials 1 and 2 , we write the resulting bimaterial curvature $r^{(C)}$ due to a change in the environmental moisture concentration $d C$ as

$\frac{1}{r^{(C)}}=\frac{\alpha_{1}^{(C)} d C_{1}-\alpha_{2}^{(C)} d C_{2}}{\frac{h}{2}+\frac{2}{h}\left(E_{1} I_{1}+E_{2} I_{2}\right)\left(\frac{1}{E_{1} h_{1}}+\frac{1}{E_{2} h_{2}}\right)}$

or

$\frac{1}{r^{(C)}}=\frac{\left[\alpha_{1}^{(C)}\left(\frac{d C_{1}}{d C}\right)-\alpha_{2}^{(C)}\left(\frac{d C_{2}}{d C}\right)\right] d C}{\frac{h}{2}+\frac{2}{h}\left(E_{1} l_{1}+E_{2} l_{2}\right)\left(\frac{1}{E_{1} h_{1}}+\frac{1}{E_{2} h_{2}}\right)}$

where $\left(d C_{1} / d C\right)$ and $\left(d C_{2} / d C\right)$ in Eq. (12b) quantify the ratio of moisture concentration changes in materials 1 and 2 vis-à-vis the moisture concentration change in the surrounding environment. Suppose $d C_{1} / d C=d C_{2} / d C=1$, Eq. (12) reduces to the form similar to Eq. (8). In order to focus on the effects of the individual material's expansion coefficient, we consider the bimaterial strips to possess equal thicknesses $\left(h_{1}=h_{2}=h / 2\right)$ and equal Young's moduli $\left(E_{1}=E_{2}\right)$, such that Eqs. (8), (11) and (12) simplify to

$$
\begin{gathered}
\frac{1}{r^{(T)}}=\frac{3}{2} \frac{1}{h}\left(\alpha_{1}^{(T)}-\alpha_{2}^{(T)}\right) d T \\
\frac{1}{r^{(P)}}=\frac{3}{2} \frac{1}{h}\left(\alpha_{1}^{(P)}-\alpha_{2}^{(P)}\right)(-d P) \\
\frac{1}{r^{(C)}}=\frac{3}{2} \frac{1}{h}\left(\alpha_{1}^{(C)} d C_{1}-\alpha_{2}^{(C)} d C_{2}\right)
\end{gathered}
$$

In modelling any effective coefficient of expansion, consideration is made to the strain induced by the environmental change. As such, reference points are to be identified for tracking relative displacements. These reference points are the center of the rigid square and the center of its nearest neighbour, as furnished in Fig. 5 .

Due to the different radii of curvatures encountered by the bimaterial strip imposed by the different types of environmental changes, the following analyses begin with modelling for the effective $C M E$, followed by reduction to the effective CTE and effective CPE for both grids. Arising from the large deflection and the corresponding finite relative displacement between the rigid squares, the usual definition of strain for infinitesimal deformation is written as an increment strain

$d \varepsilon=\frac{d L}{L}$

so as to pave a way for describing the total strain as
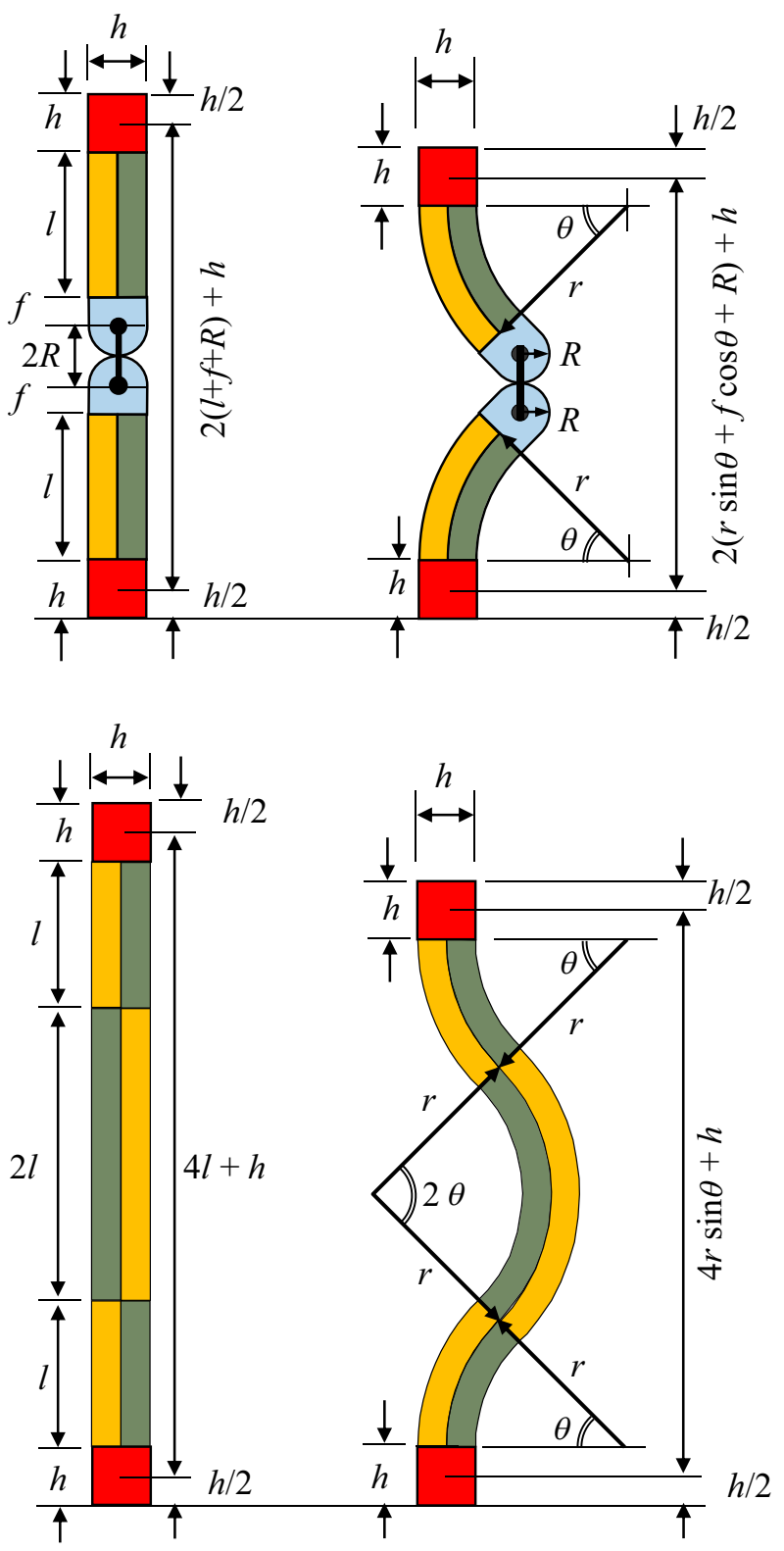

Fig. 5 Elements between two rigid blocks for the Type A grid (top) and Type B grid (bottom) in their original state (left) and deformed state (right). Reference points for relative displacements are the centers of rigid squares indicated in red. The thicknesses have been exaggerated for clarity

$\varepsilon=\int d \varepsilon=\ln \frac{L_{f}}{L_{i}}$

where $L_{i}$ and $L_{f}$ are the initial and final distances between reference points, respectively. This gives the moisture strain

$\varepsilon_{C}=\ln \frac{2\left(r^{(C)} \sin \theta+f \cos \theta+R\right)+h}{2(l+f+R)+h}$ 
for Type A (Fig. 5, top), and

$\varepsilon_{C}=\ln \frac{4 r^{(C)} \sin \theta+h}{4 l+h}$

for Type B (Fig. 5, bottom). The length of a bimaterial strip is typically 2 or 3 orders higher than its thickness, which means that $r \sim l \gg h \approx f \approx R$ such that both Eqs. (16) and (17) abridge to

$\varepsilon_{C}=\ln \left\{\frac{r^{(C)} \sin \theta}{l}\right\}$

Substituting $\theta=I / r$ and Eq. (13) into Eq. (18) gives

$\varepsilon_{C}=\ln \left\{\frac{\sin \left[\frac{3}{2} \frac{1}{h}\left(\alpha_{1}^{(C)} d C_{1}-\alpha_{2}^{(C)} d C_{2}\right)\right]}{\left[\frac{3}{2} \frac{1}{h}\left(\alpha_{1}^{(C)} d C_{1}-\alpha_{2}^{(C)} d C_{2}\right)\right]}\right\}$

With reference to Eq. (7), we have the effective CME

$\alpha^{(C)}=\frac{1}{d C} \ln \left\{\frac{\sin \left[\frac{3}{2} \frac{1}{h}\left(\alpha_{1}^{(C)} d C_{1}-\alpha_{2}^{(C)} d C_{2}\right)\right]}{\left[\frac{3}{2} \frac{1}{h}\left(\alpha_{1}^{(C)} d C_{1}-\alpha_{2}^{(C)} d C_{2}\right)\right]}\right\}$

By comparing Eq. (7) against Eqs. (2) and (4)—or by comparing Eq. (12a) against Eqs. (8) and (11) —one may infer the effective CTE and the effective compressibility as

$\alpha^{(T)}=\frac{1}{d T} \ln \left\{\frac{\sin \left[\frac{3}{2} \frac{1}{h}\left(\alpha_{1}^{(T)}-\alpha_{2}^{(T)}\right) d T\right]}{\left[\frac{3}{2} \frac{1}{h}\left(\alpha_{1}^{(T)}-\alpha_{2}^{(T)}\right) d T\right]}\right\}$

and

$\alpha^{(P)}=\frac{1}{(-d P)} \ln \left\{\frac{\sin \left[\frac{3}{2} \frac{1}{h}\left(\alpha_{1}^{(P)}-\alpha_{2}^{(P)}\right)(-d P)\right]}{\left[\frac{3}{2} \frac{1}{h}\left(\alpha_{1}^{(P)}-\alpha_{2}^{(P)}\right)(-d P)\right]}\right\}$

respectively, whereby $(-d P)$ indicates that for a conventional or positive value of compressibility, an increase in pressure results in decrease in size. As the sine function is odd, i.e. $\sin (-z)=-\sin (z)$, Eq. (22a) can also be written as

$\alpha^{(P)}=-\frac{1}{d P} \ln \left\{\frac{\sin \left[\frac{3}{2} \frac{1}{h}\left(\alpha_{1}^{(P)}-\alpha_{2}^{(P)}\right) d P\right]}{\left[\frac{3}{2} \frac{1}{h}\left(\alpha_{1}^{(P)}-\alpha_{2}^{(P)}\right) d P\right]}\right\}$

Since $|\sin z|<|z|$ except when $z=0$, it follows that $\ln \{\cdots\}<0$ in Eqs. (20) to (22). Therefore the signs of $\alpha^{(C)}$ and $\alpha^{(T)}$ are always opposite to the signs of $d C$ and $d T$, while the sign of $\alpha^{(P)}$ is always the same as that for $d P$, in consistency with the overall deformation conceptualized in Figs. 3 and 4. Having obtained models of expansion coefficients which switch signs for opposing change of environmental condition such that the Type $A$ and Type $B$ metamaterials always exhibit in-plane isotropic contraction based on the magnitude of the environmental change, an attempt in now made to specify the condition by which the deformed grid fits into the 8-pointed star. If the bimaterial deflection is insufficient, the pointed corners of the star along the diagonals are further from the center than those aligned along the vertical and horizontal axes. Beyond a certain value of bimaterial deflection, the diagonal pointed corners are nearer to the center than those lying on the axes. To fit the deformed metamaterial grid onto the 8-pointed star, the distance of the diagonal pointed corners from the center must be equal to those on the axes. This is shown from the superposition of an 8-pointed star (Fig. 6, top) onto each unit of the metamaterial (Fig. 6, middle).

Geometrical parameters $a$ and $b$ in Fig. 6 (bottom) facilitates the determination of conditions for fitting the deformed metamaterial onto the 8-pointed star. The parameter $a$ is half of the distance between the centers of two nearest rigid squares (indicated in red). Therefore, with reference to the vertical distance between the centers of the rigid squares in Fig. 5 (right),

$a=r \sin \theta+f \cos \theta+R+\frac{h}{2}$

for Type A, and

$a=2 r \sin \theta+\frac{h}{2}$

for Type B. The parameter $b$ in Fig. 6 (bottom left) corresponds to the horizontal distance component between the center of a rigid square and the outermost surface of the pillow block bearing in Fig. 5 (top right), i.e.

$b=r(1-\cos \theta)+f \sin \theta+R$

for Type A, while the same parameter in Fig. 6 (bottom right) refers to the horizontal distance component between the center of a rigid square and the maximum deflection at the mid-point of the bimaterial strip in Fig. 5 (bottom right), i.e.

$b=2 r(1-\cos \theta)$

for Type B. These give the distances from the center of each deformed grid to the grid rib intersecting the vertical and horizontal axes as

$a+b=r(1+\sin \theta-\cos \theta)+f(\sin \theta+\cos \theta)+2 R+\frac{h}{2}$ 
Fig. 6 Superposition of an 8-pointed star (top) onto a unit of the deformed metamaterials (middle), with parameters assigned for analysis (bottom)

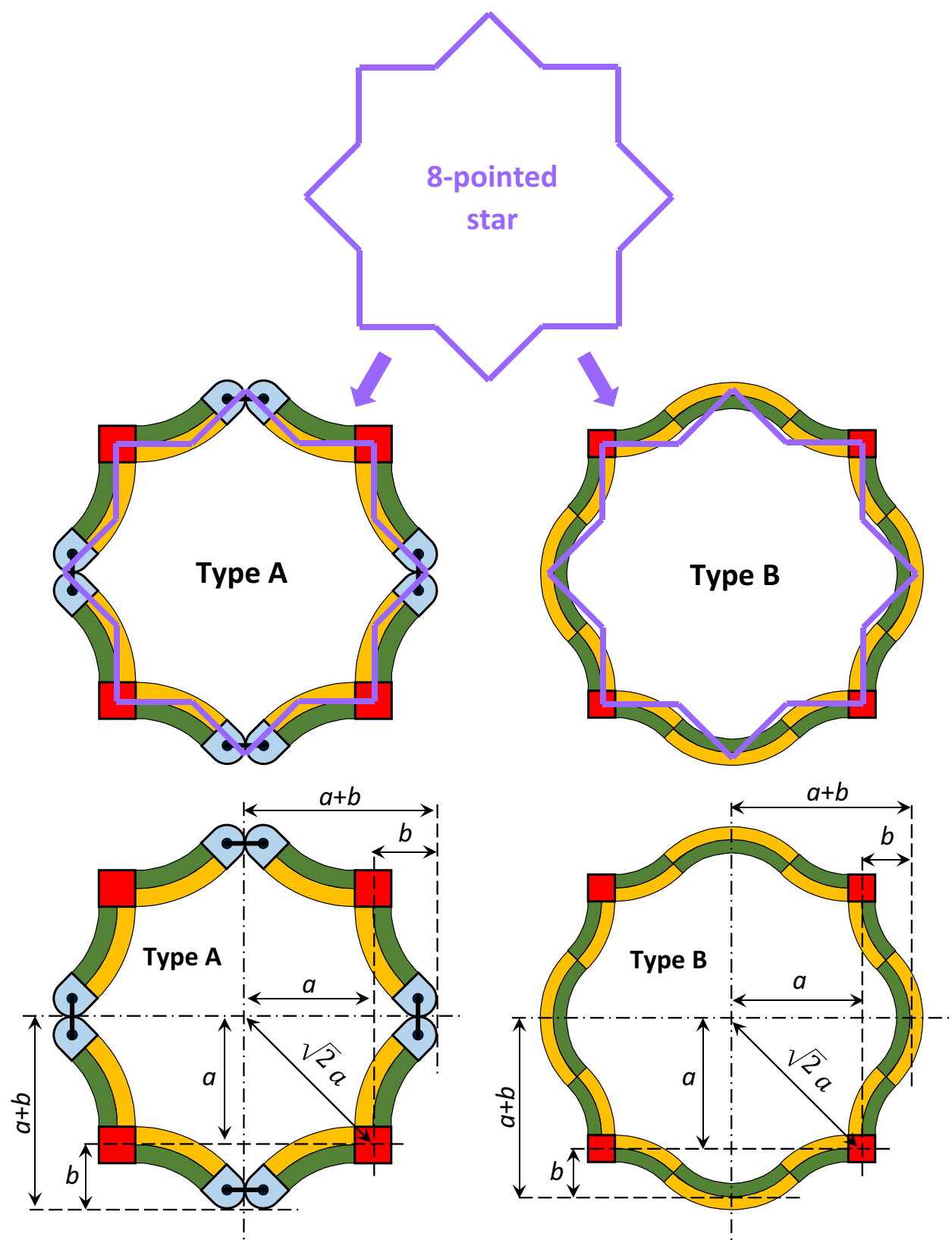

and

$a+b=2 r(1+\sin \theta-\cos \theta)+\frac{h}{2}$

for Type A and Type B, respectively. Perusal to Fig. 6 (bottom) implies that the diagonal distances between the center of the deformed grid and the center of each rigid square are the hypotenuse, i.e.

$\sqrt{2} a=\sqrt{2}(r \sin \theta+f \cos \theta+R)+\frac{h}{\sqrt{2}}$

and

$$
\sqrt{2} a=2 \sqrt{2} r \sin \theta+\frac{h}{\sqrt{2}}
$$

for metamaterials of Types $A$ and $B$, respectively. Hence to implement equidistance for all the eight points of the star from the deformed grid center, we let $a+b=\sqrt{2} a$ such that

$$
\begin{aligned}
r(1 & +\sin \theta-\cos \theta)+f(\sin \theta+\cos \theta)+2 R+\frac{h}{2} \\
& =\sqrt{2}(r \sin \theta+f \cos \theta+R)+\frac{h}{\sqrt{2}}
\end{aligned}
$$


for Type A, and

$2 r(1+\sin \theta-\cos \theta)+\frac{h}{2}=2 \sqrt{2} r \sin \theta+\frac{h}{\sqrt{2}}$

for Type B. Recall that the length of a bimaterial strip is typically 2 or 3 orders higher than its thickness, thereby implying $r \sim I \gg h \sim f \sim R$, such that Eqs. (31) and (32) simplify to a common expression

$1-\cos \theta=(\sqrt{2}-1) \sin \theta$

This relation is satisfied if $\sin \theta=\cos \theta=1 / \sqrt{2}$, i.e. the deformed metamaterials fit the 8-pointed star array geometry shown in Fig. 1 if $\theta=\pi / 4$. With reference to Eq. (18) and $l / r=\theta$, one may elegantly express the various environmental strains as

$\varepsilon=\ln \left\{\frac{\sin \theta}{l / r}\right\}=\ln \left\{\frac{\sin \theta}{\theta}\right\}$

such that substitution of $\theta=\pi / 4$ suggests that the effective strain $\varepsilon=\ln \{2 \sqrt{2} / \pi\}=-0.10501$ must be achieved to attain the desired 8-pointed star array. Furthermore, matching Eq. (34) against Eqs. (20) to (22) implies

$$
\begin{aligned}
\theta & =\frac{3}{2} \frac{I}{h}\left(\alpha_{1}^{(C)} d C_{1}-\alpha_{2}^{(C)} d C_{2}\right)=\frac{3}{2} \frac{I}{h}\left(\alpha_{1}^{(T)}-\alpha_{2}^{(T)}\right) d T \\
& =\frac{3}{2} \frac{I}{h}\left(\alpha_{1}^{(P)}-\alpha_{2}^{(P)}\right) d P
\end{aligned}
$$

which reduces to

$\alpha_{1}^{(C)} d C_{1}-\alpha_{2}^{(C)} d C_{2}=\left(\alpha_{1}^{(T)}-\alpha_{2}^{(T)}\right) d T=\left(\alpha_{1}^{(P)}-\alpha_{2}^{(P)}\right) d P=\frac{\pi}{6}\left(\frac{h}{l}\right)$

when $\theta=\pi / 4$. In other words, if properties of materials 1 and 2 of the bimaterial strip are known, then the array of 8-pointed stars can be achieved from the originally square grids of Type A and Type B metamaterial by controlling the environmental change.

\section{Results and discussion}

In order to ascertain that the assumption $f=R=h=0$ is valid, we return to Eqs. (16) and (17) to divide all terms at the numerator and denominator with $r^{(C)}$ to obtain

$\varepsilon_{C}=\ln \frac{2\left(\sin \theta+\frac{f}{l}\left(\frac{1}{r^{(C)}}\right) \cos \theta+\frac{R}{l}\left(\frac{1}{r^{(C)}}\right)\right)+\frac{h}{l}\left(\frac{1}{r^{(C)}}\right)}{2\left(\frac{1}{r^{(C)}}+\frac{f}{l}\left(\frac{1}{r^{(C)}}\right)+\frac{R}{l}\left(\frac{1}{r^{(C)}}\right)\right)+\frac{h}{l}\left(\frac{1}{r^{(C)}}\right)}$

for Type A, and
$\varepsilon_{C}=\ln \frac{4 \sin \theta+\frac{h}{l}\left(\frac{1}{r^{(C)}}\right)}{4 \frac{1}{r^{(C)}}+\frac{h}{l}\left(\frac{1}{r^{(C)}}\right)}$

for Type B, such that the substitution of $I / r^{(C)}=\theta=\pi / 4$ into Eqs. (37) and (38) leads to

$\varepsilon_{C}=\ln \frac{\frac{2 \sqrt{2}}{\pi}+\frac{1}{\sqrt{2}} \frac{f}{l}+\frac{R}{l}+\frac{1}{2} \frac{h}{l}}{1+\frac{f}{l}+\frac{R}{l}+\frac{1}{2} \frac{h}{l}}$

and

$\varepsilon_{C}=\ln \frac{\frac{2 \sqrt{2}}{\pi}+\frac{1}{4} \frac{h}{l}}{1+\frac{1}{4} \frac{h}{l}}$

respectively. Since the typical bimaterial strip aspect ratio is such that $l$ is about 2 or 3 orders higher than $h$, and perusal to Fig. 5 indicates that $R=h / 2$, we substitute $h / I=2 R / I=0.01$ into Eq. (39) to observe the effect of $f / I$ for Type A moisture strain when the 8-pointed star array is attained. Figure 7 (left) shows that, under the considered typical parameter values, the percentage error hovers around $1 \%$. Since Type B is independent from $f$ and $R$, evaluation is made on the effect of $h / l$ ratio on the moisture strain. Figure 7 (right) shows that the percentage error for the typical $h / I$ ratio falls within $0.2644 \%$. Even if the bimaterial aspect ratio is increased to the unrealistically high value of $h / I=0.1$, the percentage error for assuming $h=0$ is only $2.637 \%$. These observations validate the assumption of $f=R=h=0$ for the typical bimaterial aspect ratio.

Having demonstrated the applicability of the developed models when the typical bimaterial aspect ratio is adopted, we shall now turn our attention to the various facets of bimaterial curving in order to attain the array of 8-pointed stars under varying moisture concentration, temperature and pressure. Typically, the coefficient of moisture expansion in polymers ranges between $\alpha_{1}^{(C)}=2 \times 10^{-3}$ and $\alpha_{1}^{(C)}=5 \times 10^{-3}$ [51-54]. For the case of moisture expansion, we consider the abovementioned polymers as material 1 while material 2 is of the same polymeric material but with waterproof coating so that $d C_{2}=0$. The choice of same material ensures $E_{1}=E_{2}$ so that the simplification of Eq. (12) to the last of Eq. (13) applies. Although $\alpha_{1}^{(C)}=\alpha_{2}^{(C)}$, expansion of material 2 is inhibited due to waterproofing so that $\alpha_{2}^{(C)} d C_{2}=0$. Adopting these values for Eq. (36) gives

$d C_{1}=\frac{1}{\alpha_{1}^{(C)}} \frac{\pi}{6}\left(\frac{h}{l}\right)$

This is plotted in Fig. 8 for $2 \times 10^{-3} \leq \alpha_{1}^{(C)} \leq 5 \times 10^{-3}$ within $0 \leq h / l \leq 0.01$. 

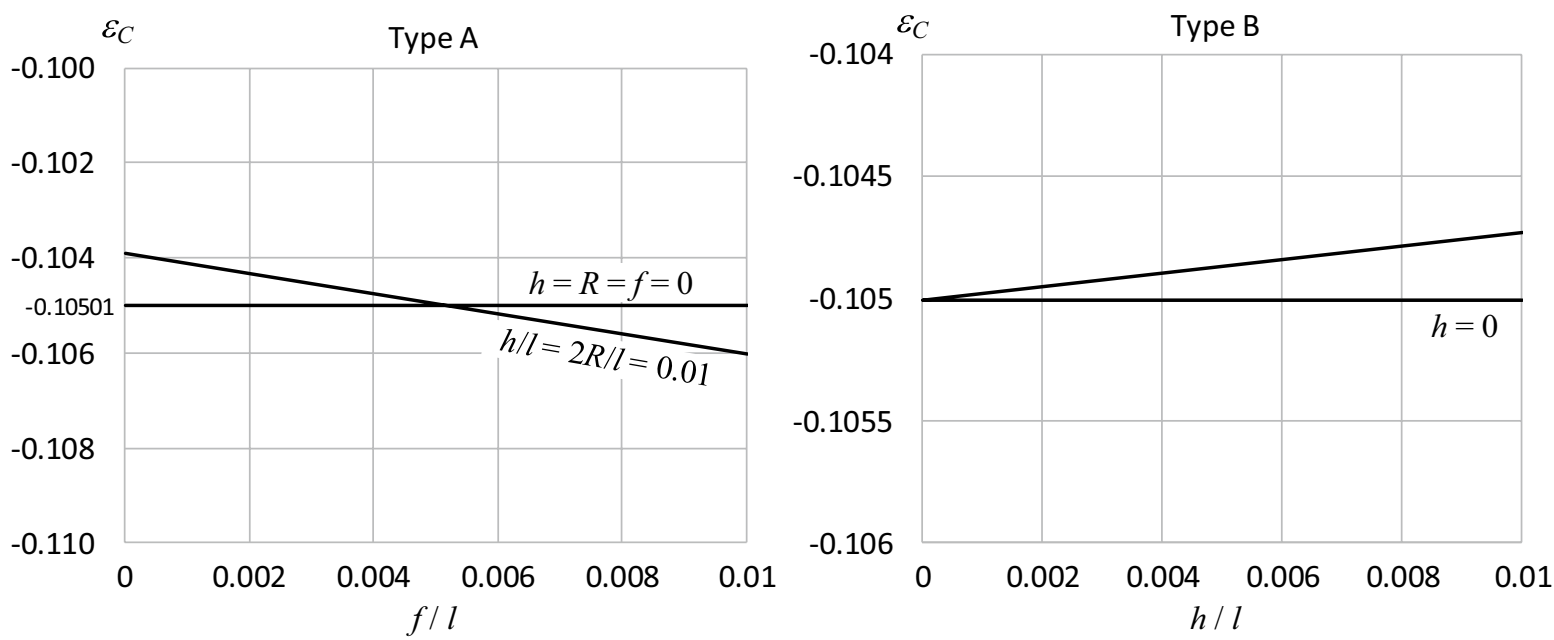

Fig. 7 Plots of moisture strain versus for $f / I$ at $h / I=2 R / I=0.01$ for Type A (left) and versus $h / I$ for Type B (right) for comparison against the simplifying assumptions of $f=R=h=0$

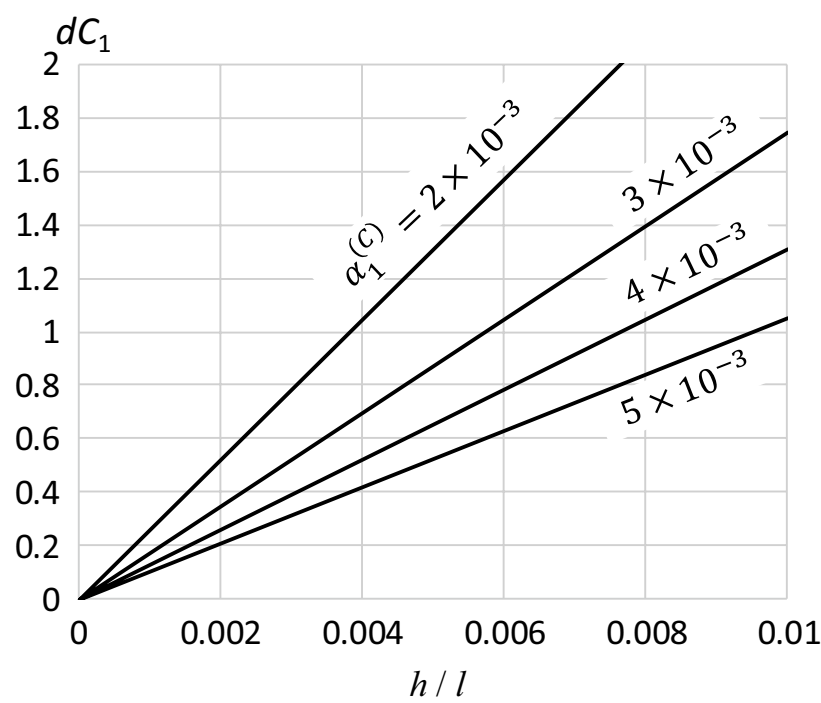

Fig. 8 Plots of required moisture absorbed into, or dissipated from, material 1 made from polymers while material 2 is of same material with waterproof coating $\left(d C_{2}=0\right)$ in order for both metamaterials Type A and Type B to attain the 8-pointed star array

For the case of thermal expansion, we consider the brass-titanium (B-T), copper-steel (C-S) and tungsten-silicon-carbide (T-SC) bimaterial pairs. The motivation for pairing of these materials can be seen from their almost equal Young's modulus $E_{1} \approx E_{2}$ but with sufficient contrast of their individual CTEs so that, instead of using Eq. (8), one is justified to use the first of Eq. (13), which is the simplified form. Their thermomechanical properties are listed in Table $2[55,56]$ and, using these properties, the required temperature change in order for both metamaterials Type A and Type B to attain the 8-pointed star array by using pairs of tungsten-silicon
Table 2 Material properties for the brass-titanium (B-T), coppersteel (C-S) and tungsten-silicon-carbide (T-SC) bimaterial pairs

\begin{tabular}{|c|c|c|}
\hline Material pairs & CTE [55] & Young's modulus [56] \\
\hline $\begin{array}{l}1=\text { Brass } \\
2=\text { Titanium }\end{array}$ & $\begin{array}{l}\alpha_{1}^{(T)}=19.0 \times 10^{-6} \mathrm{~K}^{-1} \\
\alpha_{2}^{(T)}=8.6 \times 10^{-6} \mathrm{~K}^{-1}\end{array}$ & $\begin{array}{l}E_{1}=100 \text { to } 125 \mathrm{GPa} \\
E_{2}=110.3 \mathrm{GPa}\end{array}$ \\
\hline $\begin{array}{l}1=\text { Copper } \\
2=\text { Steel }\end{array}$ & $\begin{array}{l}\alpha_{1}^{(T)}=17 \times 10^{-6} \mathrm{~K}^{-1} \\
\alpha_{2}^{(T)}=11 \text { to } 13 \times 10^{-6} \mathrm{~K}^{-1}\end{array}$ & $\begin{array}{l}E_{1}=117 \mathrm{GPa} \\
E_{2}=200 \mathrm{GPa}\end{array}$ \\
\hline $\begin{array}{l}1=\text { Tungsten } \\
2=\text { Silicon carbide }\end{array}$ & $\begin{array}{l}\alpha_{1}^{(T)}=4.50 \times 10^{-6} \mathrm{~K}^{-1} \\
\alpha_{2}^{(T)}=2.77 \times 10^{-6} \mathrm{~K}^{-1}\end{array}$ & $\begin{array}{l}E_{1}=400 \text { to } 410 \mathrm{GPa} \\
E_{2}=450 \mathrm{GPa}\end{array}$ \\
\hline
\end{tabular}

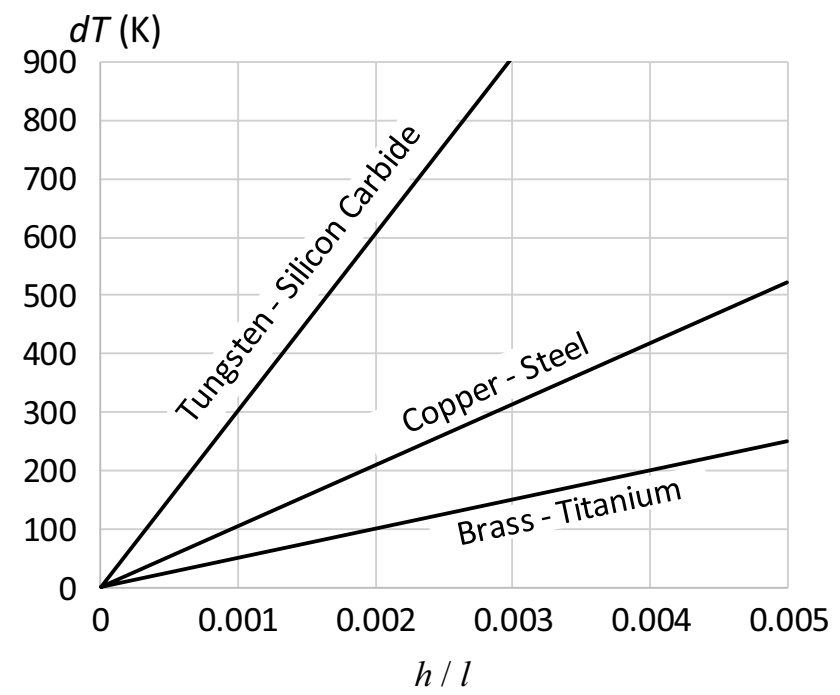

Fig. 9 Plots of required temperature change in order for both metamaterials Type A and Type B to attain the 8-pointed star array by using pairs of tungsten-silicon carbide, copper-steel and brass-titanium bimaterial strips 
carbide, copper-steel and brass-titanium bimaterial strips are plotted in Fig. 9 using Eq. (36).

For the case of compressibility, we consider the magnesium-silicon $(M-S)$, zinc-niobium ( $Z-N)$ and manganese-nickel $(M-N)$ bimaterial pairs. The motivation for selecting these pairs of materials can be seen from their almost equal Young's moduli $E_{1} \approx E_{2}$ but with ample disparity of their individual compressibilities so that, instead of employing Eq. (11), one is justified to adopt the reduced form given in the second of Eq. (13). Due to the lack of CPE data, these were converted from their bulk modulus data [57] as follows. From the definition of bulk modulus,

$K=-V \frac{d P}{d V}$

which is a reciprocal of Eq. (3) whereby $V=L^{3}$ and $d V=3 L^{2} d L$, substituting $d V / V=3 d L / L=3 \varepsilon_{p}$ into Eq. (42) gives

$K=\frac{-d P}{3 \varepsilon_{P}}$

which, upon comparison with Eq. (4), gives

$\alpha^{(P)}=\frac{1}{3 K}$

Their properties are listed in Table 3. The required pressure change in order for both metamaterials Type $A$ and Type $B$ to attain the 8-pointed star array by using pairs of magnesium-silicon, zinc-niobium and manganese-nickel strips are plotted in Fig. 10 using Eq. (36).

An explanation on Figs. 9 and 10 can now be made in the light of metamaterials concept. As metamaterials are known to demonstrate properties from their artificially designed microarchitecture, the choice of bimaterial strip arrangements shown in Fig. 3 (top) and Fig. 4 (top) not only permits bending of the straight strips into curves that approximate the Islamic design, but the exact 8-pointed star arrays-displayed Figs. 1 and 6 -are attained when the base properties of the bimaterial strips, environmental changes and bimaterial aspect ratio $h / /$ fulfil the condition set out in Eq. (36). To get the

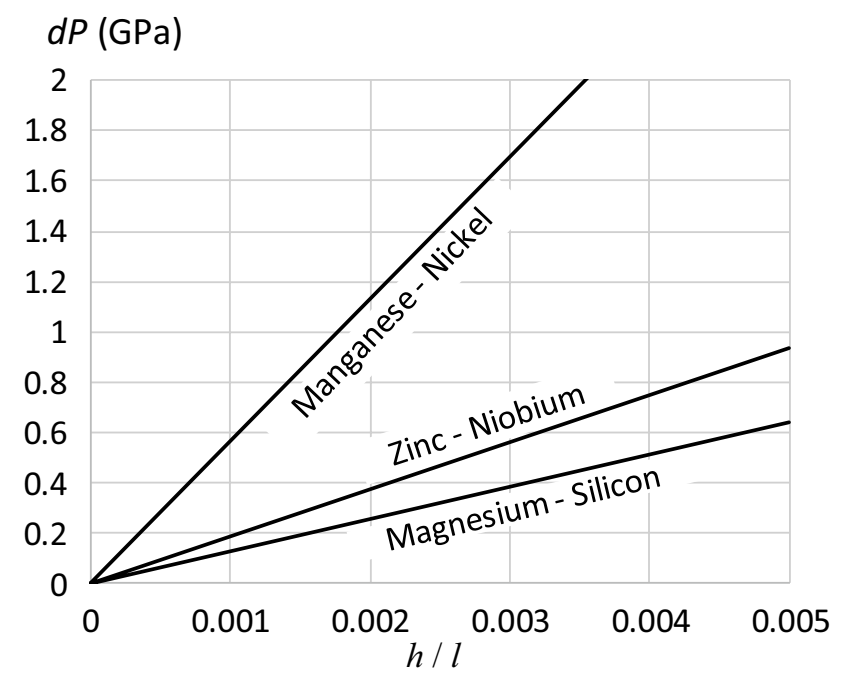

Fig. 10 Plots of required pressure change in order for both metamaterials Type A and Type B to attain the 8-pointed star array by using pairs of magnesium-silicon, zinc-niobium and manganesenickel bimaterial strips

2D array of 8-pointed stars featured in Fig. 6 in response to temperature change, one will need to refer to a relevant plot in Fig. 9 pertaining to one of the bimaterial pairs, for example. If the root mean square of the environment's temperature fluctuation is known, then the required bimaterial aspect ratio $h / I$ can be read from the horizontal axis of Fig. 9. Likewise, to obtain the 8-pointed star arrays of Fig. 6 due to pressure variation, one can peruse to a related plot in Fig. 10 corresponding to one of the bimaterial pairs, for instance. Suppose the root mean square of the environment's pressure undulation is known, then the $h / /$ ratio of the bimaterial strip can be taken from the abscissa of Fig. 10. In other words, for a given environmental variation and bimaterial pairs with known properties, the discussed Islamic motif can be achieved by choosing the slenderness of the bimaterial strip. This geometrical control of the microstructures is associated with metamaterials design concept.
Table 3 Material properties for the magnesium-silicon $(\mathrm{M}-\mathrm{S})$, zinc-niobium (Z-N) and manganese-nickel $(\mathrm{M}-\mathrm{N})$ bimaterial pairs

\begin{tabular}{llcl}
\hline Material pairs & Young's modulus [57] & Bulk modulus [57] & Compressibility \\
\hline 1 = Magnesium & $E_{1}=45 \mathrm{GPa}$ & $K_{1}=45 \mathrm{GPa}$ & $\alpha_{1}^{(P)}=7.407 \times 10^{-3} \mathrm{GPa}^{-1}$ \\
2 = Silicon & $E_{2}=47 \mathrm{GPa}$ & $K_{2}=100 \mathrm{GPa}$ & $\alpha_{2}^{(P)}=3.333 \times 10^{-3} \mathrm{GPa}^{-1}$ \\
& $E_{1}=108 \mathrm{GPa}$ & $K_{1}=70 \mathrm{GPa}$ & $\alpha_{1}^{(P)}=4.762 \times 10^{-3} \mathrm{GPa}^{-1}$ \\
1 = Zinc & $E_{2}=105 \mathrm{GPa}$ & $K_{2}=170 \mathrm{GPa}$ & $\alpha_{2}^{(P)}=1.961 \times 10^{-3} \mathrm{GPa}^{-1}$ \\
2 = Niobium & $E_{1}=198 \mathrm{GPa}$ & $K_{1}=120 \mathrm{GPa}$ & $\alpha_{1}^{(P)}=2.778 \times 10^{-3} \mathrm{GPa}^{-1}$ \\
$1=$ Manganese & $E_{2}=200 \mathrm{GPa}$ & $K_{2}=180 \mathrm{GPa}$ & $\alpha_{2}^{(P)}=1.852 \times 10^{-3} \mathrm{GPa}^{-1}$ \\
$2=$ Nickel & & & \\
\end{tabular}




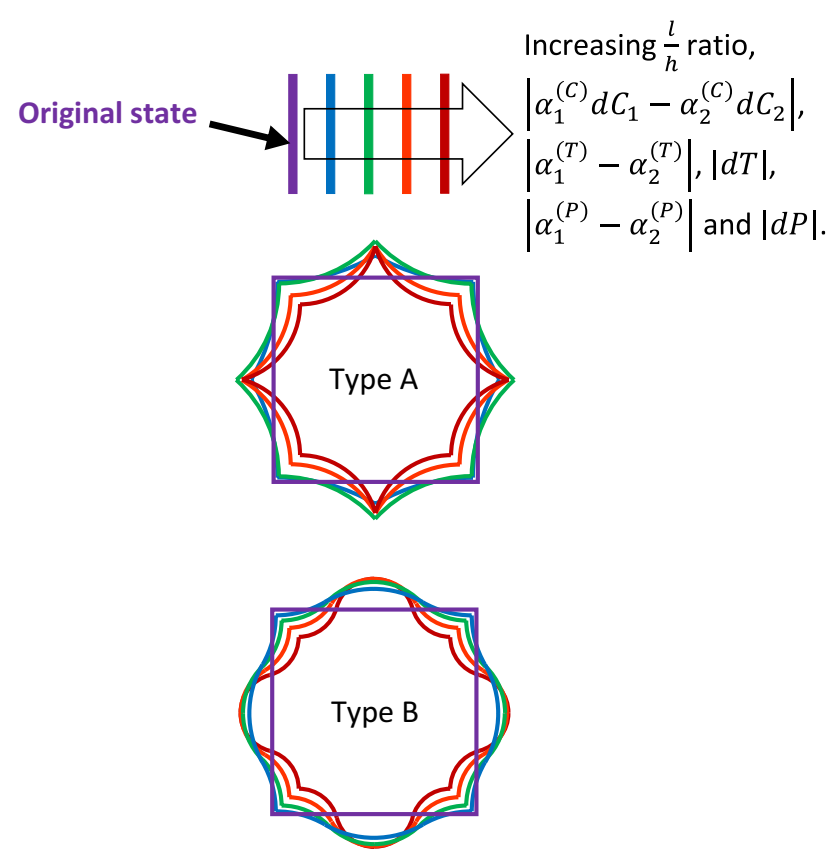

Fig. 11 Responses of Type A and Type B units with variation of geometrical, material and environmental parameters

Finally, a summary can be made on the responses of both the Type A and Type B units for each variable, and these are categorized into 3 groups:

(a) The geometrical parameters, such as the Type $A$ and Type $B$ arrangements, the bimaterial thickness $h$ and a segment of its length $I$, and in the case of Type $A$ the geometrical parameters of the connecting parts $f$ and $R$;

(b) The material parameters, such as the linear coefficients of moisture expansion $\alpha_{1}^{(C)}$ and $\alpha_{2}^{(C)}$, linear coefficients of thermal expansion $\alpha_{1}^{(T)}$ and $\alpha_{2}^{(T)}$, and linear compressibilities $\alpha_{1}^{(P)}$ and $\alpha_{2}^{(P)}$ of the bimaterial phases 1 and 2; and

(c) The environmental parameters, such as the changes of moisture concentration $d C_{1}$ and $d C_{2}$, temperature $d T$, and pressure $d P$ on bimaterial phases 1 and 2 .

The radius of curvature $r$ formed is not included as it is a function of the parameters listed in (a) to (c).

With reference to Fig. 11 for the morphing of the Type A and Type B cell shapes in response to the parameters, the extent of deformation is facilitated by the following groups of parameters:

(i) Increase in the bimaterial aspect ratio for each segment $I / h$ (purely geometrical parameters); (ii) Increase in the differences between expansion coefficients $\left|\alpha_{1}^{(T)}-\alpha_{2}^{(T)}\right|$ and $\left|\alpha_{1}^{(P)}-\alpha_{2}^{(P)}\right|$ (purely material parameters);

(iii) Increase the environmental variations $|d T|$ and $|d P|$ (purely environmental parameters); and

(iv) Increase in the moisture strains difference between the bimaterial phases $\left|\alpha_{1}^{(C)} d C_{1}-\alpha_{2}^{(C)} d C_{2}\right|$ (combined material and environmental parameters).

The parameters are described in their magnitudes in order to include negative values. With reference to the diagonal array of 8-pointed stars with alternating crossshaped cells-such as those shown in Fig. 2 (bottom), Fig. 3 (bottom) or Fig. 4 (bottom) -it is the adjacent neighbors that form the 8-pointed stars when the signs are negative. Effects from the geometrical parameters of the connecting parts $f$ and $R$ are negligible for the practical range of $h / l$ with $I \gg h \sim f \sim R$, and as a consequence the effects of selecting either Type $A$ or Type $B$ is also insignificant.

\section{Conclusions}

Neither positive nor negative expansion coefficients are fully advantageous across a wide range of structural applications that are exposed to environmental fluctuations. In some cases, it is advantageous for materials to possess duality in material properties so as to take advantage of negative behavior under a change in environmental condition as well as conventional behavior when the condition reverses. Unlike recent works on sign switching materials properties, which are only interesting from technological viewpoint, the current 2D metamaterial is additionally aesthetic. Originally in the form of square grids, both types considered herein deform into arrays of 8-pointed stars, which are known to be prevalent in Islamic art and architecture. These have been made achievable by the use of bimaterial strips that are arranged in a certain manner. The metamaterials concept is adopted by the arrangements of the bimaterial strips in a certain manner within each repeating units, and by controlling the bimaterial thickness-to-length $h / /$ ratio.

\section{Compliance with ethical standards}

Conflict of interest The corresponding author states that there is no conflict of interest. 


\section{References}

1. Tümkaya MA, Karaaslan M, Sabah C (2018) Metamaterial-based high efficiency portable sensor application for determining branded and unbranded fuel oil. Bull Mater Sci 41:91

2. Abdulkarim YI, Deng L, Awl HN, Muhammadsharif FF, Altintas O, Karaaslan M, Luo H (2020) Design of a broadband coplanar waveguide-fed antenna incorporating organic solar cells with $100 \%$ insolation for Ku band satellite communication. Materials $13: 142$

3. Ozdemir E, Akgol O, Alkurt FO, Karaaslan M, Abdulkarim Yl, Deng $L$ (2020) Mutual coupling reduction of cross-dipole antenna for base stations by using a neural network approach. Appl Sci 10:378

4. Abdulkarim Yl, Deng L, Karaaslan M, Altintas O, Awl HN, Muhammadsharif FF, Liao C, Unal E, Luo H (2020) Novel metamaterialsbased hypersensitized liquid sensor integrating omega-shaped resonator with microstrip transmission line. Sensors 20:943

5. Dalgac S, Bakır M, Karadag F, Karaaslan M, Akgol O, Unal E, Sabah C (2020) Microfluidic sensor applications by using chiral metamaterial. Mod Phys Lett B 34:2050031

6. Evans KE (1991) Auxetic polymers: a new range of materials. Endeavour 15:170-174

7. Alderson A, Alderson KL (2007) Auxetic materials. J Aerosp Eng 221:565-575

8. Liu Y, Hu H (2010) A review on auxetic structures and polymeric materials. Sci Res Essays 5:1052-1063

9. Greaves GN, Greer AL, Lakes RS, Rouxel T (2011) Poisson's ratio and modern materials. Nat Mater 10:823-837

10. Carneiro VH, Meireles J, Puga H (2013) Auxetic materials-a review. Mater Sci Poland 31:561-571

11. Novak N, Vesenjak M, Ren Z (2016) Auxetic cellular materials-a review Strojniški Vestnik. J Mech Eng 62:485-493

12. Saxena KK, Das R, Calius EP (2016) Three decades of auxetics research-materials with negative Poisson's ratio: a review. Adv Eng Mater 18:1847-1870

13. Jiang JW, Kim SY, Park HS (2016) Auxetic nanomaterials: Recent progress and future development. Appl Phys Rev 3:041101

14. Lim TC (2017) Analogies across auxetic models based on deformation mechanism. Phys Status Solidi RRL 11:1600440

15. Kolken HMA, Zadpoor AA (2017) Auxetic mechanical metamaterials. RSC Adv 7:5111-5129

16. Park HS, Kim SY (2017) A perspective on auxetic nanomaterials. Nano Converg 4:10

17. Lakes RS (2017) Negative-poisson's-ratio materials: auxetic solids. Ann Rev Mater Res 47:63-81

18. Papadopoulou A, Laucks J, Tibbits S (2017) Auxetic materials in design and architecture. Nat Rev Mater 2:17078

19. Duncan O, Shepherd T, Moroney C, Foster L, Venkatraman PD, Winwood K, Allen T, Alderson A (2018) Review of auxetic materials for sports applications: expanding options in comfort and protection. Appl Sci 8:941

20. Ren X, Das R, Tran P, Ngo TD, Xie YM (2018) Auxetic metamaterials and structures: a review. Smart Mater Struct 27:023001

21. Lim TC (2015) Auxetic materials and structures. Springer, Singapore

22. Hu H, Zhang M, Liu Y (2019) Auxetic textiles. Elsevier, Duxford

23. Lim TC (2005) Anisotropic and negative thermal expansion behavior in a cellular microstructure. J Mater Sci 40:3275-3277

24. Miller W, Smith CW, MacKenzie DS, Evans KE (2009) Negative thermal expansion: a review. J Mater Sci 44:5441-5451

25. Lim TC (2012) Negative thermal expansion structures constructed from positive thermal expansion trusses. J Mater Sci 47:368-373
26. Ng CK, Saxena KK, Das R, Flores EIS (2017) On the anisotropic and negative thermal expansion from dual-material re-entranttype cellular metamaterials. J Mater Sci 52:8999-9912

27. Ai L, Gao XL (2017) Metamaterials with negative Poisson's ratio and non-positive thermal expansion. Compos Struct 162:70-84

28. Lim TC (2017) Auxetic and negative thermal expansion structure based on interconnected array of rings and sliding rods. Phys Status Solidi B 254:1600775

29. Wei K, Peng Y, Wang K, Duan S, Yang X, Wen W (2018) Three dimensional lightweight lattice structures with large positive, zero and negative thermal expansion. Compos Struct 188:287-296

30. Li Y, Chen Y, Li T, Cao S, Wang L (2018) Hoberman-sphereinspired lattice metamaterials with tunable negative thermal expansion. Compos Struct 189:586-597

31. Oddone V, Wimpory RC, Reich S (2019) Understanding the negative thermal expansion in planar graphite-metal composites. J Mater Sci 54:1267-1274

32. Gatt R, Caruana-Gauci R, Grima JN (2013) Negative linear compressibility: Giant response. Nat Mater 12:182-183

33. Magos-Palasyuk E, Fijalkowski KJ, Palasyuk T (2016) Chemically driven negative linear compressibility in sodium amidoborane, $\mathrm{Na}\left(\mathrm{NH}_{2} \mathrm{BH}_{3}\right)$. Sci Rep 6:28745

34. Lim TC (2017) 2D structures exhibiting negative area compressibility. Phys Status Solidi B 254:1600682

35. Caruana-Gauci R, Degabriele EP, Attard D, Grima JN (2018) Auxetic metamaterials inspired from wine-rack. J Mater Sci 53:5079-5091

36. Feng G, Zhang WX, Dong L, Li W, Cai W, Wei W, Ji L, Lin Z, Lu P (2019) Negative area compressibility of a hydrogen-bonded two-dimensional material. Chem Sci 10:1309-1315

37. Degabriele EP, Attard D, Grima-Cornish JN, Caruana-Gauci R, Gatt R, Evans KE, Grima JN (2019) On the compressibility properties of the wine-rack-like carbon allotropes and related poly(phenylacetylene) systems. Phys Status Solidi B 256:1800572

38. Lim TC (2018) A negative hygroscopic expansion material. Mater Sci Forum 928:277-282

39. Lim TC (2019) A reinforced kite-shaped microstructure with negative linear and area hygrothermal expansions. Key Eng Mater 803:272-277

40. Lim TC (2019) Negative environmental expansion for interconnected array of rings and sliding rods. Phys Status Solidi B 256:1800032

41. Lim TC (2019) Composite microstructures with Poisson's ratio sign switching upon stress reversal. Compos Struct 209:34-44

42. Lim TC (2019) Metamaterials with Poisson's ratio sign toggling by means of microstructural duality. SN Appl Sci 1:176

43. Lim TC (2019) A composite metamaterial with sign switchable elastic and hygrothermal properties induced by stress direction and environmental change reversals. Compos Struct 220:185-193

44. Lim TC (2019) A 2D auxetikos system based on interconnected shurikens. SN Appl Sci 1:1383

45. Lim TC (2019) 2D metamaterial with in-plane positive and negative thermal expansion and thermal shearing based on interconnected alternating bimaterials. Mater Res Express 6:115804

46. Lim TC (2019) A class of shape-shifting composite metamaterial honeycomb structures with thermally-adaptive Poisson's ratio signs. Compos Struct 226:111256

47. Lim TC (2019) Composite metamaterial with sign-switchable coefficients of hygroscopic, thermal and pressure expansions. Adv Compos Hybrid Mater 2:657-669 
48. Lim TC (2020) Metacomposite with auxetic and in situ sign reversible thermal expansivity upon temperature fluctuation. Compos Commun 19:30-36

49. Rafsanjani A, Pasini D (2016) Bistable auxetic mechanical metamaterials inspired by ancient geometric motifs. Extreme Mech Lett 9:291-216

50. Timoshenko SP (1925) Analysis of bi-metal thermostats. J Opt Soc Am 11:233-235

51. Adams DF, Monib MM (1980) Moisture expansion and thermal expansion coefficients of a polymer-matrix composite material. In: Lenoe EM, Oplinger DW, Burke JJ (eds) Fibrous composites in structural design. Plenum Press, New York, pp 819-830

52. Adamson MJ (1980) Thermal expansion and swelling of cured epoxy resin used in graphite/epoxy composite materials. J Mater Sci 15:1736-1745

53. Cairns DS, Adams DF (1984) Moisture and thermal expansion properties of unidirectional composite materials and the epoxy matrix. In: Springer GS (ed) Environmental effects on composite materials, 2nd edn. Technomic Publishing Company, Lancaster, pp 300-325
54. Weitsman YJ (2000) Effects of fluids on polymeric composites-a review. In: Kelly A, Zweben C (eds) Comprehensive composite materials, 2nd edn. Elsevier, Amsterdam, pp 369-401

55. Wikipedia-Thermal expansion https://en.wikipedia.org/ wiki/Thermal_expansion\#Coefficient_of_thermal_expansion. Accessed Feb 24, 2020

56. Wikipedia-Young's modulus https://en.wikipedia.org/wiki/ Young\%27s_modulus Accessed Feb 24, 2020

57. Wikipedia-Elastic properties of the elements (data page) https ://en.wikipedia.org/wiki/Elastic_properties_of_the_elements_ (data_page) Accessed Feb 24, 2020

Publisher's Note Springer Nature remains neutral with regard to jurisdictional claims in published maps and institutional affiliations. 